\title{
ins
}

\section{Drought-tolerant rice germplasm developed from an Oryza officinalis transformation- competent artificial chromosome clone}

\author{
R. Liu*, H.H. Zhang*, Z.X. Chen, M.Q. Shahid, X.L. Fu and X.D. Liu \\ College of Agriculture, South China Agricultural University, Guangzhou, \\ Guangdong, China
}

*These authors contributed equally to this study.

Corresponding author: X.D. Liu

E-mail: xdliu@scau.edu.cn

Genet. Mol. Res. 14 (4): 13667-13678 (2015)

Received April 23, 2015

Accepted July 14, 2015

Published October 28, 2015

DOI http://dx.doi.org/10.4238/2015.October.28.29

\begin{abstract}
Oryza officinalis has proven to be a natural gene reservoir for the improvement of domesticated rice as it carries many desirable traits; however, the transfer of elite genes to cultivated rice by conventional hybridization has been a challenge for rice breeders. In this study, the conserved sequence of plant stress-related NAC transcription factors was selected as a probe to screen the $O$. officinalis genomic transformation-competent artificial chromosome library by Southern blot; 11 positive transformation-competent artificial chromosome clones were subsequently detected. By Agrobacterium-mediated transformation, an indica rice variety, Huajingxian 74 (HJX74), was transformed with a TAC clone harboring a NAC gene-positive genomic fragment from $O$. officinalis. Molecular analysis revealed that the $O$. officinalis genomic fragment was integrated into the genome of HJX74. The transgenic lines exhibited high tolerance to drought stress. Our results demonstrate that the introduction of stress-related transformation-competent artificial chromosome clones, coupled with a transgenic validation approach, is an
\end{abstract}


effective method of transferring agronomically important genes from $O$. officinalis to cultivated rice.

Key words: Agrobacterium tumefaciens; Drought stress; Oryza officinalis; Gene transformation; Wild rice

\section{INTRODUCTION}

Rice (Oryza sativa L.) accounts for the majority of food consumed by the world's human population. To meet the requirements of an increasing global human population and climate change, many high yield, high quality, and stress-tolerant rice varieties have been developed by different breeding strategies, by transferring the desirable traits of wild rice into cultivated rice. The genus Oryza contains 20 wild rice species, which are classified into 10 distinct genome types (AA, $\mathrm{BB}, \mathrm{CC}, \mathrm{EE}, \mathrm{FF}, \mathrm{GG}, \mathrm{BBCC}, \mathrm{CCDD}, \mathrm{HHJJ}$, and HHKK). Wild rice has many valuable features, including tolerance to different biotic and abiotic stressors; therefore, wild rice is considered a good genetic resource for rice improvement (Zhong et al., 1995; Wei et al., 2010; Liu et al., 2011; Tong et al., 2011). O. officinalis $(2 n=24, C C)$ was originally distributed throughout South China, and is a perennial species with resistance to bacterial blight, rice blast, the brown planthopper (Nilaparvata lugens), and leaf rollers, is salt- and cold-tolerant, and has a high protein content, so is an important germplasm for rice breeding (Zhong et al., 1995).

Many studies have mined novel resistance genes from O. officinalis (Shin and Katayama, 1979; Jena and Khush, 1990; Jena et al., 1992; Yan et al., 1996; Huang et al., 2001; Du et al., 2009). Although progress on using desirable $O$. officinalis genes has been made, the efficiency of transferring elite $O$. officinalis genes into cultivated rice is still low, because of interspecific reproductive isolation and the simultaneous integration of undesirable traits by conventional hybridization. Interspecific and intergeneric reproduction barriers could be overcome by improving transgenic technology. Efficient gene-fragment transfer has been achieved in several studies (Hiei et al., 1994; Rashid et al., 1996). The genetic transformation of large chromosomal fragments of wild rice into cultivated rice has many advantages, including the avoidance of interspecific reproduction barrier bottlenecks, the construction of whole-genome mutant libraries for the discovery of lines for rice breeding, and the identification of favorable genes by sub-cloning and sequencing (Cao, 2005). The first genomic library of $O$. officinalis was constructed with a plant transformationcompetent binary bacterial artificial chromosome (BIBAC), and the candidate clones of target genes isolated from the library were directly used to transform Japonica rice variety $\mathrm{H} 1493$ by an Agrobacterium-mediated transformation method (He, 2003). Another transformation-competent artificial chromosome (TAC) vector (Liu et al., 2002) was used to construct an O. officinalis genomic library (unpublished data), and we have transferred several clones of this TAC library into indica and japonica rice varieties (Wang et al., 2010; Liu et al., 2014). Both studies confirmed the feasibility of transferring agronomically important genes from wild rice species to cultivated rice by largefragment transformation.

Plants are subjected to various biotic and abiotic stresses during the process of growth and development, and drought is one of the main environmental stressors that affect agricultural productivity (Khattab et al., 2014). Plants have evolved different self-defense mechanisms against different environmental stressors. Transcription factors play important roles in the regulation of stress response-related gene expression. Five plant-specific transcription factor families (MYB, 
BZIP, WRKY, AP2/ERF, and NAC) related to biotic and abiotic stressors have been extensively studied (Lata et al., 2011). NAC transcription factors are plant-specific proteins that play an important role in plant development and abiotic and/or biotic stress responses (Olsen et al., 2005). An NAC transcription factor has been identified for dehydration stress (gene RD26) in Arabidopsis (Yamaguchi-Shinozaki et al., 1992). In rice, which is a monocot model plant, the expression of several members of the NAC family, including SNAC1, OsNAC6/SNAC2, OsNAC5, OsNAC10, and ONAC45, is induced by drought, salt, and abscisic acid (ABA) treatment. Functional analysis has indicated that these NAC genes play important roles in resistance to drought and salt stress (Hu et al., 2006; Jeong et al., 2010). Arabidopsis NAC genes have similar functions as rice NAC genes (Lu et al., 2007; Wu et al., 2009). Expression levels of rice OsNAC19 increase after infection with the blast pathogen Magnaporthe oryzae and exposure to the plant hormones methyl jasmonate and ABA (Lin et al., 2007). Arabidopsis ATAF1 and ATAF2 (NAC transcription factors) are negative regulators of defense responses against necrotrophic fungal and bacterial pathogens (Delessert et al., 2005; Wang et al., 2009).

In this study, we selected the conserved sequence of stress-related NAC genes as a probe to screen the $O$. officinalis genomic TAC library, and candidate transformation-competent artificial chromosome clones were directly transferred into indica rice by Agrobacterium-mediated transformation. The responses of transgenic lines to drought stress were tested by polyethylene glycol (PEG) treatment at the bud stage. The results indicate that genomic library screening and the functional analysis of transgenic validation is an effective method of mining desirable genes from O. officinalis.

\section{MATERIAL AND METHODS}

\section{TAC library screening}

The O. officinalis TAC library was kindly donated by Y. Liu of the South China Agricultural University, and consists of 20,631 clones with an average insert size of $30 \mathrm{~kb}$ (range 15-50 kb) and is stored in 54 culture-plates with 384-hole each plate. Two successive Southern blots were conducted to screen the TAC library. For the first hybridization screening, 14 pieces of high-density hybridization membrane were prepared by gridding each clone on a Hybond- $\mathrm{N}^{+}$nylon membrane (Amersham) in a $3 \times 3$ array. To avoid false positives, each clone was gridded in double spots, which had DNA corresponding to four 384-well library plates (Liu et al., 2014).

\section{Hybridization membrane preparation}

The hybridization membranes with gridded clones were transferred onto lysogeny broth plates with $1.5 \%$ agar, $25 \mu \mathrm{g} / \mu \mathrm{L}$ kanamycin, and $0.5 \mathrm{mM}$ isopropyl $\beta$-D-1-thiogalactopyranoside, and incubated at $37^{\circ} \mathrm{C}$ for $17-18 \mathrm{~h}$. The colony membranes were placed on $3 \mathrm{M}^{\mathrm{Tm}}$ filter paper that was soaked with a buffer containing $5 \%$ sodium dodecyl sulfate (SDS) and $0.2 X$ saline sodium citrate (SSC), and then both the hybridization membranes and the filter paper were completely dried in a microwave oven for $3 \mathrm{~min}$. The hybridization membranes were rinsed with a washing solution $(0.5 \%$ SDS and 0.2X SSC) and dried again. Finally, the hybridization membranes were wrapped in plastic film and stored at $4^{\circ} \mathrm{C}$. For the second hybridization screening, three hybridization membranes were prepared with the same procedure as described above, except that each of the first-detected clones was gridded in quadratic spots. 


\section{Hybridization probe preparation}

The conserved sequence of the NAC transcription family was amplified by polymerase chain reaction (PCR) and used as a probe to screen the TAC library for the isolation and identification of positive clones. The NAC gene primers 5'-ACCTGGTTTCCGCTTTCAC-3' and 5'-TGGTGCCGTTTTCGTTG-3' were used to amplify the cDNA of a Japonica cultivar, Nipponbare. The PCR amplification procedure was as follows: pre-denaturation at $94^{\circ} \mathrm{C}$ for $5 \mathrm{~min}$, then $94^{\circ} \mathrm{C}$ for $30 \mathrm{~s}, 58^{\circ} \mathrm{C}$ for $30 \mathrm{~s}, 72^{\circ} \mathrm{C}$ for $30 \mathrm{~s}$ for $35 \mathrm{cycles}$, and a final extension at $72^{\circ} \mathrm{C}$ for $10 \mathrm{~min}$. For the first hybridization screening, the probe was labeled with [ $\left.\alpha^{-P^{32}}\right]$ dCTP, according to the Random Primer DNA Labeling Kit protocol (TaKaRa, Dalian, China).

\section{Hybridization (Southern blot) and selection of positive clones}

The colony membranes with transformation-competent artificial chromosome clones were pre-hybridized with the hybridization solution and then hybridized overnight with the $\alpha$ - $\mathrm{P}^{32}$-labeled probe. The membranes were washed with the washing solution and then wrapped in plastic film. The radioactive signal was detected with a phosphor screen and analyzed using the analytical software Quantity One ${ }^{\circledast}$ (Bio-Rad). Clones that exhibited signals on the symmetrical spots were considered candidate positive clones. For the second hybridization screening, the probe was labeled with digoxigenin, and Southern blot was conducted according to the DIG High Prime DNA Labeling and Detection Starter Kit I protocol (Roche).

Each positive clone was further identified by PCR using NAC primers. Amplifications were conducted using the following program: initial denaturation at $94^{\circ} \mathrm{C}$ for $5 \mathrm{~min}$, followed by 35 cycles of denaturation at $94^{\circ} \mathrm{C}$ for $30 \mathrm{~s}$, annealing at $58^{\circ} \mathrm{C}$ for $30 \mathrm{~s}$, and extension at $72^{\circ} \mathrm{C}$ for $30 \mathrm{~s}$, followed by a final extension for $10 \mathrm{~min}$ at $72^{\circ} \mathrm{C}$.

\section{Genetic transformation and molecular identification of transgenic plants}

A transformation-competent artificial chromosome clone (49N-A10) was selected from the library and introduced into Agrobacterium tumefaciens EHA105 by electroporation. The Agrobacterium cells harboring the TAC plasmid were used to transform scutellar calli derived from mature embryos of an indica rice variety, Huajingxian 74 (HJX74), according to Wang et al. (2010) with a minor modification. Potential transformed rice calli were transferred onto a regeneration Murashige and Skoog medium supplemented with $50 \mathrm{mg} / \mathrm{L}$ hygromycin. The putative transgenic plants were identified by PCR. The DNA was extracted according to the sodium dodecyl sulfate simple extraction method (Yang et al 2012). A pair of primers was designed according to the HPT element sequence of a TAC vector, and consisted of Hpt-forward 5'-TCCGGAGCCTCCGCTCGAAGTAG-3' and Hpt-reverse 5'-CTGAACTCACCGCGACGTCGTC-3'. The PCR amplification procedure contained 35 cycles, including denaturation at $94^{\circ} \mathrm{C}$ for $30 \mathrm{~s}$, annealing at $56^{\circ} \mathrm{C}$ for $90 \mathrm{~s}$, and extension at $72^{\circ} \mathrm{C}$ for $90 \mathrm{~s}$.

\section{Evaluation of agronomic and drought-tolerant traits of transgenic plants}

Eleven agronomic traits were investigated: plant height, flag leaf length, flag leaf width, spike neck length, spike neck thickness, seed-setting rate, grain length, grain width, and 1000-seed weight. 
To evaluate the drought tolerance of the transgenic plants, rice seeds were surfacesterilized in 5\% sodium hypochloride for 20 min and washed in distilled water, and then germinated in $0,10,15$, or $20 \%$ PEG-6000 solutions. The observation and evaluation of drought tolerance was conducted with two repetitions. Seed germination rate was investigated at the 2nd, 4th, 6th, and 8th day after treatment. Bud, root, and coleoptile lengths were measured at the 8th day after treatment, and the radicle length to plumule length ratio was calculated. The germination index, germination stress index, relative bud length, relative coleoptile length, relative root length, the relative radicle length and plumule length ratio at different concentrations were also calculated. The drought response index is the ratio of the value measured under stress to that of the control.

\section{RESULTS}

\section{Isolation and identification of candidate transformation-competent artificial chromosome clones}

In order to identify desirable genes from the $\mathrm{O}$. officinalis TAC library, we selected the conserved sequence of the NAC transcription family as a probe by PCR amplification. A pair of primers was designed, rice cDNA was used as a template, and the targeted fragment was amplified that had exactly the same sequence as predicted. For the primary library screening, the PCR product was labeled with $\alpha-\mathrm{P}^{32}$ and used as a probe in the Southern blot, which produced 679 positive clones from the TAC library (Figure 1a). Southern blotting was conducted for false positives and was followed by hybridization screening with a probe labeled with digoxigenin, and the screened clones were gridded in quadratic spots; 24 positive clones were subsequently detected (Figure 1b). In order to transform indica rice with targeted transformation-competent artificial chromosome clones, PCR with NAC-specific primers was conducted, and 11 out of 24 positive clones exhibited a distinct band (Figure 1c). Therefore, these 11 positive transformationcompetent artificial chromosome clones could be used for genetic transformation in indica rice.

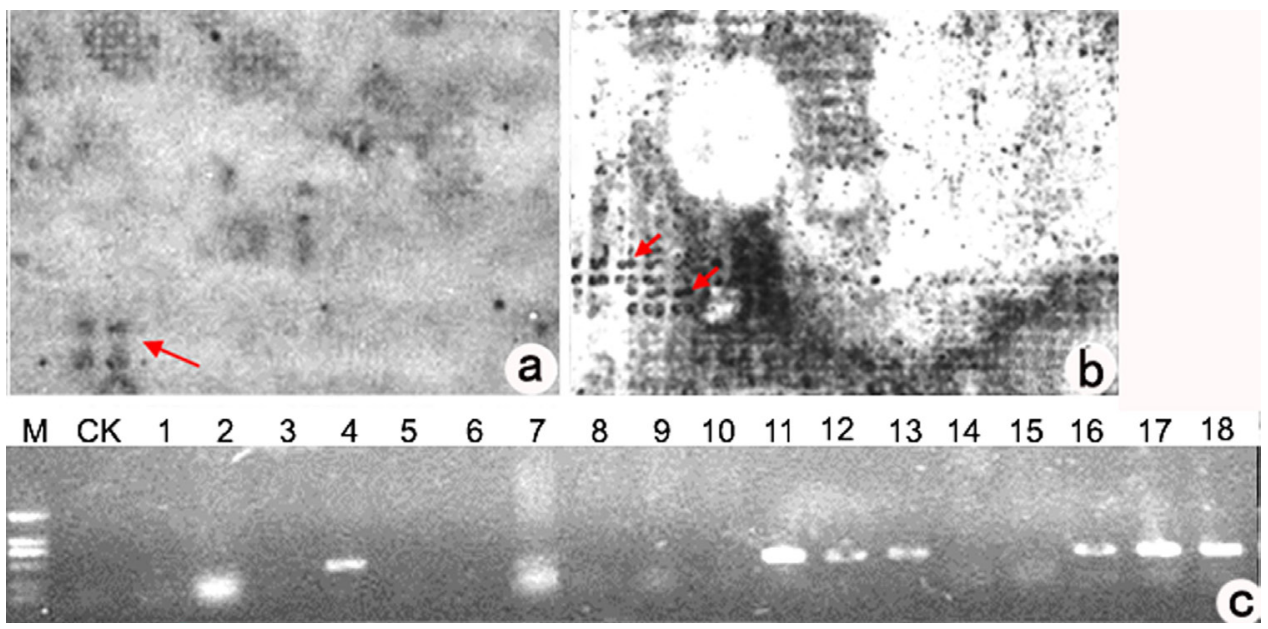

Figure 1. Hybridization screening of TAC library of $O$. officinalis. a. The scanning images of the primary hybridization screening. The arrow indicated hybridization spot. b. The scanning images of the secondary hybridization screening. The arrow indicated hybridization spot. c. Confirmation of candidate positive clones by PCR with NAC-specific primers. 


\section{Genetic transformation and molecular identification of transgenic plants}

A transformation-competent artificial chromosome clone $(49 \mathrm{~N}-\mathrm{A} 10)$ that carried a 10 kb genomic sequence checked by Hind $W$ digestion (data not shown) was selected for genetic transformation. By electroporation, the plasmid of 49N-A10 was introduced into $A$. tumefaciens EHA105, which was used to transform scutellar calli derived from mature embryos of the indica rice variety HJX74. Consequently, 17 independent transgenic lines were regenerated (Figure 2af). A PCR with primers specific to the HPT gene was conducted, which confirmed that the total transgenic lines had integrated the exogenous $O$. officinalis genomic segment (Figure $2 \mathrm{~g}$ ).

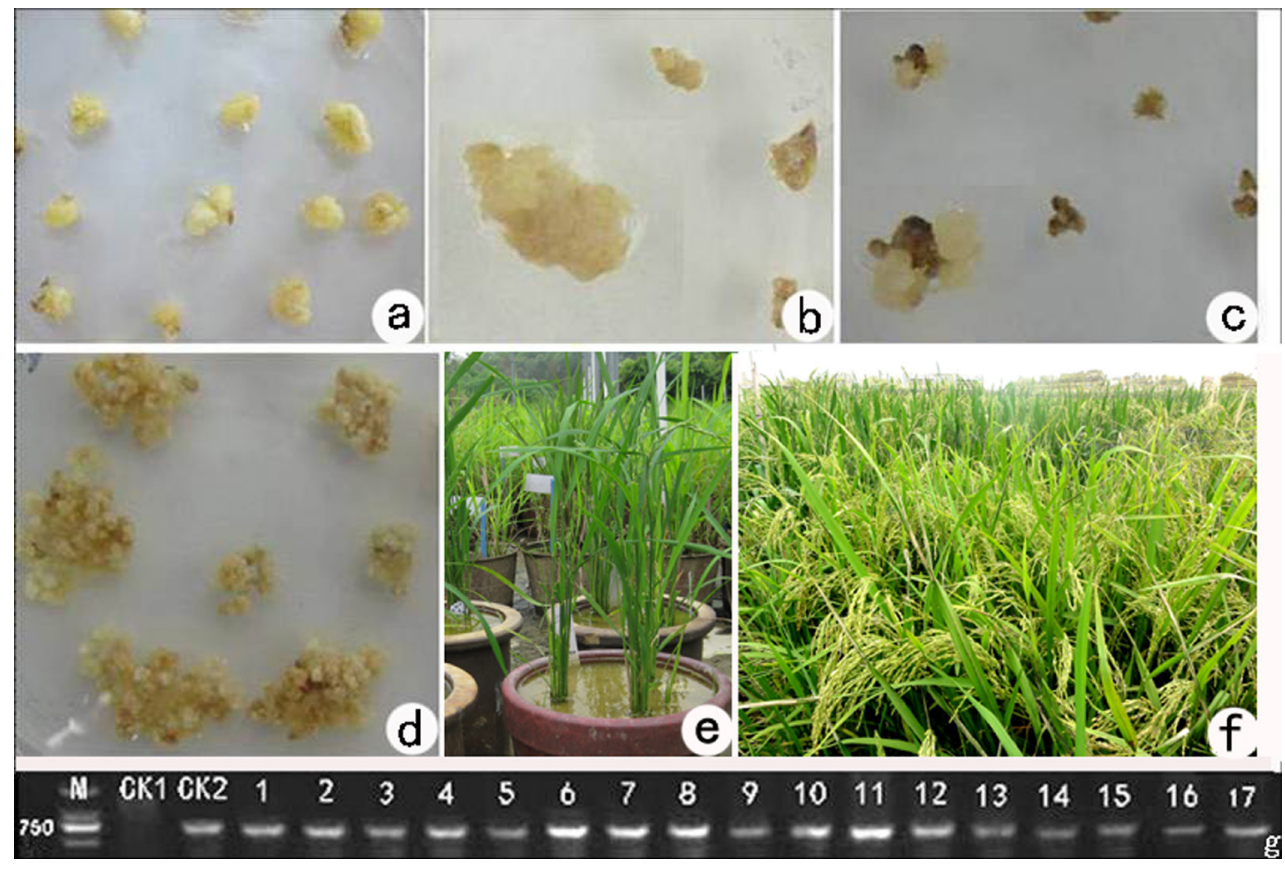

Figure 2. The process of the genetic transformation of HJX74. a. and $\mathbf{b}$. The scutellarcalli derived from mature embryos of indica rice variety HJX74. c. The scutellarcalli transformed by Agrobacterium tumefaciens. $\mathbf{d}$. The scutellarcalli were regenerated. e. The transgenic lines. f. Drought tolerant transgenic rice was planted in field. g. 17 different transgenic lines $\left(T_{0}\right)$ were identified by PCR.

\section{Evaluation of agronomic and drought-tolerant traits of transgenic plants}

Four of the 17 independent putative transgenic plants $\left(T_{0}\right)$ were sterile. Three hundred and sixty-three transgenic plants $\left(T_{1}\right)$ were derived from the 13 fertile transgenic plants and planted in the field. PCR amplification with HPT gene-specific primers generated distinct bands from the genomic DNA of 184 transgenic plants, further confirming that the $O$. officinalis genomic fragment had integrated into the HJX74 genome. These 184 transformed plants exhibited differences to the recipient variety, particularly in plant height and seed-setting rate. However, no statistical difference between the varieties was detected in the other agronomic traits (Table 1). 
Table 1. Comparison of agronomic characters of the transgenic plants and the recipient parent HJX74.

\begin{tabular}{lccccccccc}
\hline Name & $\begin{array}{c}\text { Plant height } \\
(\mathrm{cm})\end{array}$ & $\begin{array}{c}\text { Flag leaf } \\
\text { length }(\mathrm{cm})\end{array}$ & $\begin{array}{c}\text { Flag leaf } \\
\text { width }(\mathrm{cm})\end{array}$ & $\begin{array}{c}\text { Spike neck } \\
\text { length }(\mathrm{cm})\end{array}$ & $\begin{array}{c}\text { Spike neck } \\
\text { thickness }(\mathrm{mm})\end{array}$ & $\begin{array}{c}\text { Seed setting } \\
\text { rate }(\%)\end{array}$ & $\begin{array}{c}\text { Grain length } \\
(\mathrm{mm})\end{array}$ & $\begin{array}{c}\text { Grain width } \\
(\mathrm{mm})\end{array}$ & $\begin{array}{c}\text { 1000- seed } \\
\text { weight }(\mathrm{g})\end{array}$ \\
\hline $\mathrm{HJX74}$ & 102.1 & 33.7 & 2.02 & 0.97 & 2.69 & 95 & 8.39 & 2.70 & 21.79 \\
$\mathrm{~T}_{1}$ & $96.2^{\star *}$ & 32.1 & 1.87 & 1.96 & 2.39 & $85^{\star *}$ & 8.41 & 2.66 & 19.45 \\
\hline
\end{tabular}

**Significantly different at 0.01 probability level.

The drought resistance of the 10 transgenic plants was tested. The seed germination rate and seed germination index of the transgenic and wild-type plants tended to decrease with increasing concentrations of PEG-6000 (Table 2 and Figure 3). All of the transgenic plants tested exhibited a higher seed germination index value than HJX74 at $10 \%$ osmotic stress. However, only five transgenic plants exhibited a higher seed germination index value than HJX74 at $15 \%$ osmotic stress, and the seed germination index values of all of the materials tested were lower than that of the recipient parent at $20 \%$ osmotic stress (Table 2 and Figure 3 ). We further investigated the drought stress index for bud length, coleoptile length, root length, and root-toshoot ratio at three concentrations of osmotic stress eight days after germination (Table 3 and Figure 4). Four transgenic lines, N16-132, N14-3, N2-49, and N2-7, had higher drought stress index values for bud length, coleoptile length, root length, and root-to-shoot ratio than HJX74 at three concentrations. The coleoptile length of all of the materials tested was lower than that of the recipient parent at three concentrations of drought stress. Five lines, N16-132, N2-34, N243, N2-49, and N16-153, had higher root drought stress index values than HJX74. Two lines, N16-132 and N2-43, had higher drought stress index values for root-to-shoot ratio than HJX74. These results demonstrate that the line N16-132 exhibited greater drought tolerance than the recipient parent.

Table 2. Seed germination rate of the transformed materials of $\mathrm{T} 2$ under three different concentrations of PEG stress.

\begin{tabular}{|c|c|c|c|c|c|c|c|c|c|c|c|c|c|c|c|c|c|c|c|}
\hline \multirow[t]{2}{*}{ Name } & \multicolumn{4}{|c|}{0 (CK) } & \multicolumn{4}{|c|}{$10 \%$} & \multicolumn{4}{|c|}{$15 \%$} & \multicolumn{4}{|c|}{$20 \%$} & \multicolumn{3}{|c|}{$\begin{array}{l}\text { Seed germination } \\
\text { index }\end{array}$} \\
\hline & $2 d^{1}$ & $4 d$ & $6 d$ & $8 d$ & $2 d$ & $4 d$ & $6 d$ & $8 d$ & $2 d$ & $4 d$ & $6 d$ & $8 d$ & $2 d$ & $4 d$ & $6 d$ & $8 d$ & $10 \%$ & $15 \%$ & $20 \%$ \\
\hline HJX74 & 96.7 & 100.0 & 100.0 & 100.0 & 10.6 & 77.2 & 96.7 & 98.3 & 26.7 & 81.7 & 98.3 & 98.3 & 26.7 & 83.3 & 98.3 & 98.3 & 40.3 & 48.5 & 48.9 \\
\hline N16-132 & 96.7 & 100.0 & 100.0 & 100.0 & 76.7 & 100.0 & 100.0 & 100.0 & 20.0 & 93.3 & 93.3 & 98.3 & 15.0 & 61.7 & 81.7 & 88.3 & 68.0 & 49.1 & 37.3 \\
\hline N2-49 & 69.0 & 100.0 & 100.0 & 100.0 & 33.3 & 80.0 & 96.7 & 96.7 & 6.7 & 85.0 & 96.7 & 96.7 & 20.0 & 61.7 & 86.7 & 88.3 & 49.8 & 42.9 & 39.5 \\
\hline N2-7 & 96.7 & 100.0 & 100.0 & 100.0 & 78.3 & 98.3 & 98.3 & 98.3 & 40.0 & 90.0 & 95.0 & 95.0 & 21.7 & 78.3 & 91.7 & 91.7 & 67.8 & 53.7 & 44.8 \\
\hline N2-34 & 93.3 & 100.0 & 100.0 & 100.0 & 66.7 & 98.3 & 100.0 & 100.0 & 18.3 & 76.7 & 96.7 & 100.0 & 18.3 & 50.0 & 86.7 & 95.0 & 64.6 & 44.8 & 36.9 \\
\hline N2-43 & 93.3 & 100.0 & 100.0 & 100.0 & 21.7 & 95.0 & 96.7 & 90.0 & 10.0 & 73.3 & 90.0 & 90.0 & 16.7 & 50.0 & 65.0 & 75.0 & 49.8 & 39.8 & 31.6 \\
\hline N14-12 & 93.3 & 100.0 & 100.0 & 100.0 & 45.0 & 95.0 & 96.7 & 96.7 & 65.0 & 86.7 & 95.0 & 95.0 & 18.3 & 46.7 & 80.0 & 86.7 & 56.6 & 60.4 & 34.5 \\
\hline N14-46 & 96.7 & 100.0 & 100.0 & 100.0 & 48.3 & 100.0 & 100.0 & 100.0 & 36.7 & 96.7 & 98.3 & 98.3 & 18.3 & 50.0 & 80.0 & 86.7 & 59.5 & 54.9 & 35.3 \\
\hline N14-34 & 86.7 & 86.7 & 93.3 & 93.3 & 65.0 & 96.7 & 96.7 & 96.7 & 16.7 & 61.7 & 86.7 & 91.7 & 18.3 & 51.7 & 86.7 & 91.7 & 63.0 & 38.8 & 37.0 \\
\hline N14-3 & 100.0 & 100.0 & 100.0 & 100.0 & 66.7 & 96.7 & 95.0 & 95.0 & 31.7 & 80.0 & 95.0 & 95.0 & 26.7 & 70.0 & 86.7 & 90.0 & 63.1 & 48.9 & 43.5 \\
\hline N16-153 & 96.7 & 96.7 & 96.7 & 96.7 & 46.7 & 96.7 & 98.3 & 98.3 & 23.3 & 93.3 & 100.0 & 100.0 & 20.0 & 56.7 & 76.7 & 85.0 & 57.9 & 50.5 & 36.6 \\
\hline
\end{tabular}

\section{DISCUSSION}

The utilization of desirable genes from $\mathrm{O}$. officinalis consists of breeding an aneuploidy monosomic alien addition line (MAAL), constructing an introgression line, and obtaining individual elite traits (genes). Shin and Katayama (1979) first reported an entire set of aneuploidy MAAL plants, and each of the MAALs had a single chromosome from the $\mathrm{C}$ genome by conventional 


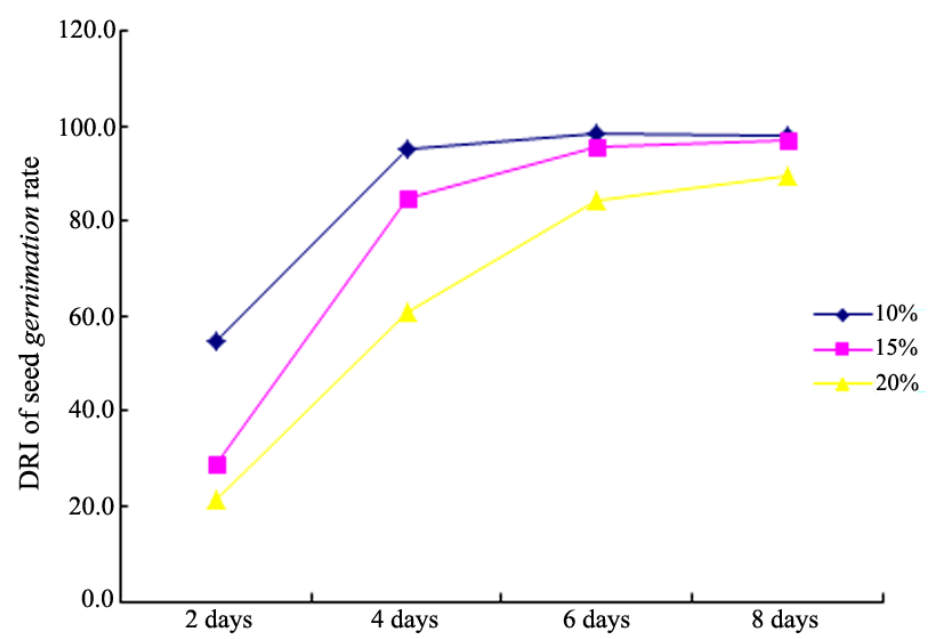

Figure 3. DRI (drought response index) of germination rate during different time periods under different concentrations of osmotic stress solution (10, 15 and $20 \%$ Polyethylene glycol).

Table 3. Drought response index for bud length, coleoptile length, root length, ratio of root length to bud length under different concentration osmotic stress solution.

\begin{tabular}{|c|c|c|c|c|c|c|c|c|c|c|c|c|c|c|c|c|}
\hline \multirow[t]{2}{*}{ Name } & \multicolumn{4}{|c|}{$\mathrm{DRI}^{1}$ of bud length } & \multicolumn{4}{|c|}{ DRI of coleoptile length } & \multicolumn{4}{|c|}{ DRI of root length } & \multicolumn{4}{|c|}{ DRI of root to shoot ratio } \\
\hline & $10 \%$ & $15 \%$ & $20 \%$ & Average & $10 \%$ & $15 \%$ & $20 \%$ & Average & $10 \%$ & $15 \%$ & $20 \%$ & Average & $10 \%$ & $15 \%$ & $20 \%$ & Averag \\
\hline & & & & & 156 & 150 & & & & & & & & & & \\
\hline & & & & & & & & & & & & & & & & \\
\hline & & & & & & & & & & & & & & & & \\
\hline & & & & & & & 100 & & & & & & & & & \\
\hline & & & & & & 126 & & & & & & & & & & \\
\hline & & & & & & & & & & & & & & & & \\
\hline & & 75.25 & 40.26 & & & & & & 432.5 & 186.88 & 166.25 & & 369.15 & & & 343.47 \\
\hline N14-34 & 107.43 & 70.29 & 32.29 & & 119.46 & 105.31 & 61.74 & & 138.7 & 82.38 & 80.08 & & 129.11 & & & 164.78 \\
\hline & 78.74 & 75.0 & 52.3 & 68.68 & 113.14 & 113.14 & 77.37 & 101.22 & 133.5 & 169.94 & 236.42 & 179.96 & 169.59 & 226.59 & 452.05 & 282.7 \\
\hline & 101.72 & & 38.68 & 67.33 & 65.06 & & & & 256.9 & 132.68 & 143.14 & & 252.52 & 215.37 & 370.04 & 279.3 \\
\hline N14-12 & 103.85 & 107.31 & 25.38 & 78.85 & 106.57 & 94.89 & 65.69 & 89.05 & 285.0 & 235.34 & 66.17 & 195.49 & 274.41 & 219.31 & 260.65 & 251.46 \\
\hline
\end{tabular}

${ }^{1} \mathrm{DRI}$ is "drought response index".

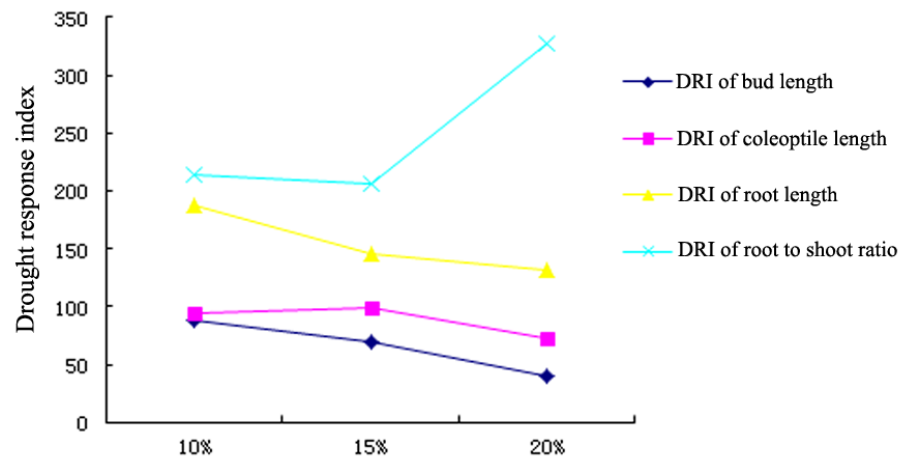

Concentration of osmotic stress solution

Figure 4. DRI (drought response index) of bud length, coleoptile length, root length and root to shoot ratio under different concentrations of Polyethylene glycol (osmotic stress solution). 
hybridization. However, they were unable to detect the additional single chromosome that the $O$. officinalis MAAL plant carries, because of the unavailability of trisomics in cultivated rice. Twelve MAAL plants were obtained by the conventional method from three cultivated rice varieties that carried the additional chromosomes of 0 . officinalis, and 11 single gene-controlled traits were transferred into cultivated rice. This indicated that genes that confer resistance to the brown planthopper and white-backed planthopper (Sogatella furcifera) are located on chromosome 6 of O. sativa (Jena and Khush, 1990). Tan et al. (2005) also produced rice MAALs containing an individual chromosome of $\mathrm{O}$. officinalis, and studied syntonic associations between the A genomes and $\mathrm{C}$ genomes using restriction fragment length polymorphism markers. Jena et al. (1992) derived 52 introgression lines from the hybrids $\left(\mathrm{BC}_{2} \mathrm{~F}_{8}\right)$ of two cultivated rice and five $\mathrm{O}$. officinalis lines. The integrated fragments of the 4th, 10th, and 12th chromosomes of $O$. officinalis are associated with resistance to the brown planthopper. The O. officinalis brown planthopper resistance gene(s) has also been transferred into two rice varieties by artificial hybridization (Yan et al., 1996). Qbp1 and Qbp2, which confer resistance to the brown planthopper, were introduced into rice chromosomes by crossing cultivated rice with $O$. officinalis (Huang et al., 2001). Bph14 was the first map-cloned gene from O. officinalis, and encodes the CC-NB-LRR protein (Du et al., 2009).

In this study, we investigated the large-insert genomic library of the non-AA genome wild rice species $O$. officinalis, in order to discover desirable genes and produce transgenic plants with high-quality traits by transgenic validation. Large-insert genomic libraries are usually used in the generation of chromosome contigs in physical mapping, and the isolation of genes by map-based cloning (Ariyadasa and Stein, 2012). To achieve the goal of this study, an O. officinalis genomic TAC library was screened to identify clones that contained stress-related NAC transcription factor genes. Agrobacterium-mediated transformation was used to generate transgenic indica plants, and evaluation of the transgenic lines was conducted by subjecting them to drought stress; the results confirmed that the transgenic plants exhibited an appreciable level of tolerance against drought stress. Our results demonstrate that this approach is an effective and alternative way of exploiting the desirable genes of $O$. officinalis. These results are consistent with those of Liu et al. (2014), who also found Agrobacterium-mediated transformation a useful technique to transfer genes from wild to cultivated rice genotypes. In rice, the NAC transcription factor family consists of 75, 140, or 151 members, depending on the bioinformatics method employed (Ooka et al., 2003; Nuruzzaman et al., 2010). The CC genome of $O$. officinalis is much larger than the AA genome of O. sativa (Uozu et al., 1997); therefore, O. officinalis might contain more NAC transcription factors than $O$. sativa. In this study, three different screening methods were successively used to detect positive NAC-related transformation-competent artificial chromosome clones. Firstly, Southern blot with the isotope $\mathrm{P}^{32}$ produced 679 positive transformation-competent artificial chromosome clones, which is more than the number of NAC transcription factors in $O$. sativa (163 in indica rice and 186 in Japonica rice, http://planttfdb.cbi.edu.cn/). Therefore, many positive transformation-competent artificial chromosome clones could be false positives when conducting Southern blot with $\mathrm{P}^{32}$. The 679 transformation-competent artificial chromosome clones detected were rescreened and 24 clones were found. This is fewer than the number of NAC transcription factors in rice, possibly because of using the Southern blot method with a DIG-labeled probe. Eleven transformation-competent artificial chromosome clones were identified as candidate clones by the primers, and screening three times confirmed that the candidate clones were NAC-specific factors for transformation.

Regarding wild rice, many studies have constructed large-insert genomic libraries, including 12 BAC libraries representing 10 genomes of Oryza species (Wing et al., 2005; Ammiraju 
et al., 2006), a BIBAC library of O. officinalis (He, 2003), TAC libraries of cultivated rice (Liu et al., 2002) and O. officinalis (unpublished data), and a BAC library of O. rufipogon (Li et al., 2008). These genomic libraries facilitate research into the comparative genetic mapping of Oryza species, gene cloning, and genome sequencing. The direct transformation of BAC clones that carry a large insert of wild rice DNA to mine valuable genes is not possible, because a BAC vector has not been genetically modified for replication in $A$. tumefaciens. Both BIBAC and TAC vectors are capable of replicating in both Escherichia coli and $A$. tumefaciens, and can be used to transform a large insert of DNA directly into the plant genome by transgenic technology (Hamilton, 1997; Liu et al., 1999, 2002). He (2003) reported that a BIBAC clone with a 120-kb O. officinalis DNA insert was successfully transferred into the Japonica rice genome, but the stress responses of the transgenic plants were not tested. In this study, an NAC-specific transformation-competent artificial chromosome clone of $\mathrm{O}$. officinalis was transformed into the indica rice genome and the droughtstress tolerance of the transgenic lines was tested; they exhibited a good tolerance to drought stress. Therefore, the direct transformation of a transformable genomic library, coupled with stressrelated clone screening, is an effective method of identifying and using desirable genes in wild rice.

\section{Conflicts of interest}

The authors declare no conflict of interest.

\section{ACKNOWLEDGMENTS}

We thank Y. Liu of the South China Agricultural University for providing the $O$. officinalis TAC library, and are grateful for the assistance of L. Wang and S. Yu. Research supported by the Guangdong Provincial Key Natural Science Foundation (\#10251064201000001 and \#2014A030311042 to X.D. Liu), Guangdong provincial key platform of University and major research project (Natural Science) - characteristic innovation project [Yue-JK2014 (65) to X.D. Liu], the Special Fund for Agro-Scientific Research in the Public Interest (\#201003021 to X.L. Fu) and the Guangdong science and technology program (\#2014A030304055 to X.D. Liu).

\section{REFERENCES}

Ammiraju JS, Luo M, Goicoechea JL, Wang W, et al. (2006). The Oryza bacterial artificial chromosome library resource: construction and analysis of 12 deep-coverage large-insert BAC libraries that represent the 10 genome types of the genus Oryza. Genome Res. 16: 140-147.

Ariyadasa R and Stein N (2012). Advances in BAC-based physical mapping and map integration strategies in plants. J. Biomed. Biotechnol. Doi: 10.1155/2012/184854.

Cao ML (2005). Strategies on exploring the favorable genes of wild rice and developing super hybrid rice based on wholegenome knock-in mutant library. Mol. Plant Breed. 3: 869-876.

Delessert C, Kazan K, Wilson IW, Van Der Straeten D, et al. (2005). The transcription factor ATAF2 represses the expression of pathogenesis-related genes in Arabidopsis. Plant J. 43: 745-757.

Du B, Zhang W, Liu B, Hu J, et al. (2009). Identification and characterization of Bph14, a gene conferring resistance to brown planthopper in rice. Proc. Natl. Acad. Sci. U. S. A. 106: 22163-22168.

Hamilton CM (1997). A binary-BAC system for plant transformation with high-molecular-weight DNA. Gene 200: $107-116$.

He RF (2003). Construction of a genomic library of Oryza Officinalis and transformation of large DNA fragments. PhD thesis, Wuhan University, Wuhan, China.

Hiei Y, Ohta S, Komari T and Kumasiro T (1994). Efficient transformation of rice mediated by Agrobacterium and sequence analysis of the boundaries of the T-DNA. Plant J. 6: 271-282. 
Hu H, Dai M, Yao J, Xiao B, et al. (2006). Overexpressing a NAM, ATAF, and CUC (NAC) transcription factor enhances drought resistance and salt tolerance in rice. Proc. Natl. Acad. Sci. U. S. A. 103: 12987-12992.

Huang Z, He G, Shu L, Li X, et al. (2001). Identification and mapping of two brown planthopper resistance genes in rice. Theor. Appl. Genet. 102: 929-934.

Jena KK and Khush GS (1990). Introgression of genes from Oryza officinalis Well ex Watt to cultivated rice, O. sativa L. Theor. Appl. Genet. 80: 737-745.

Jena KK, Khush GS and Kochert G (1992). RFLP analysis of rice (Oryza sativa L.) introgression lines. Theor. Appl. Genet. 84: 608-616

Jeong JS, Kim KS, Baek KH, Jung H, et al. (2010). Root-specific expression of OsNAC10 improves drought tolerance and grain yield in rice under field drought conditions. Plant Physiol. 153: 185-197.

Khattab HI, Emam MA, Emam MM, Helal NM, et al. (2014). Effect of selenium and silicon on transcription factors NAC5 and DREB2A involved in drought-responsive gene expression in rice. Biol. Plant 58: 265-273.

Lata C, Yadav A and Prasad M (2011). Role of plant transcription factors in abiotic stress tolerance. In: Abiotic stress response in plants - physiological, biochemical and genetic perspectives (Arun S, ed.). InTech Open, 269-296.

Li X, Tan LB, Huang H, Zhu Z, et al. (2008). Construction of a bacterial artificial chromosome (BAC) library of common wild rice (Oryza rufipogon Griff.) for map-based cloning of genes selected during the domestication of rice. Biotechnol. Lett. 30: 555-561.

Lin R, Zhao W, Meng X, Wang M, et al. (2007). Rice gene OsNAC19 encodes a novel NAC-domain transcription factor and responds to infection by Magnaporthe grisea. Plant Sci. 172: 120-130.

Liu B, Li JQ, Liu XD, Shahid MQ, et al. (2011). Identification of neutral genes at pollen sterility loci Sd and Se of cultivated rice (Oryza sativa) with wild rice (O. rufipogon) origin. Genet. Mol. Res. 10: 3435-3445.

Liu YG, Liu H, Chen L, Qiu W, et al. (2002). Development of new transformation-competent artificial chromosome vectors and rice genomic libraries for efficient gene cloning. Gene 282: 247-255.

Liu YG, Shirano Y, Fukaki H, Yanai Y, et al. (1999). Complementation of plant mutants with large genomic DNA fragments by a transformation-competent artificial chromosome vector accelerates positional cloning. Proc. Natl. Acad. Sci. U. S. A. 96: 6535-6540.

Liu R, Zhang HH, Chen ZX, Shahid MQ, et al. (2014). Development of drought -tolerant rice germplasm by screening and transforming TAC clones of Oryza officinalis Wall. Sci. Agric. Sin. 47: 1445-1457.

Lu PL, Chen NZ, An R, Su Z, et al. (2007). A novel drought-inducible gene, ATAF1, encodes a NAC family protein that negatively regulates the expression of stress responsive genes in Arabidopsis. Plant Mol. Biol. 63: 289-305.

Nuruzzaman M, Manimekalai R, Sharoni AM, Satoh K, et al. (2010). Genome-wide analysis of NAC transcription factor family in rice. Gene 465: 30-44.

Olsen AN, Ernst HA, Leggio LL and Skriver K (2005). NAC transcription factors: structurally distinct, functionally diverse. Trends Plant Sci. 10: 79-87.

Ooka H, Satoh K, Doi K, Nagata T, et al. (2003). Comprehensive analysis of NAC family genes in Oryza sativa and Arabidopsis thaliana. DNA Res. 10: 239-247.

Rashid H, Yokoi S, Toriyama K and Hinata K (1996). Transgenic plant production mediated by Agrobacterium in indica rice. Plant Cell Rep. 15: 727-730.

Shin YB and Katayama T (1979). Cytological studies on the genus Oryza. XI, Alien addition lines of O. sativa with single chromosomes of $\mathrm{O}$. officinalis. Jpn. J. Genet. 54: 1-10.

Takasaki H, Maruyama K, Kidokoro S, Ito Y, et al. (2010). The abiotic stress-responsive NAC-type transcription factor OsNAC5 regulates stress-inducible genes and stress tolerance in rice. Mol. Genet. Genome 284: 173-183.

Tan GX, Jin HJ, Li G, He RF, et al. (2005). Production and characterization of a complete set of individual chromosome additions from Oryza officinalis to Oryza sativa using RFLP and GISH analyses. Theor. Appl. Genet. 111: 1585-1595.

Tong JF, Li YH, Yang YX, Shahid MQ, et al. (2011). Molecular evolution of rice $S_{5}{ }^{n}$ and functional comparison among different sequences. Chin. Sci. Bull. 56: 2016-2024.

Uozu S, Ikehashi H, Ohmido N, Ohtsubo H, et al. (1997). Repetitive sequences: cause for variation in genome size and chromosome morphology in the genus Oryza. Plant Mol. Biol. 35: 791-799.

Wang N, Chen ZX, Liu R, Jiang YJ, et al. (2010). Preliminary study of transformation system of indica rice with TAC clones containing large genomic sequences of Oryza officinalis Wall. Plant Physiol. Commun. 46: 217-222.

Wang X, Vindhya BM, Basnayake S, Zhang HJ, et al. (2009). The Arabidopsis ATAF1, a NAC transcription factor, is a negative regulator of defense responses against necrotrophic fungal and bacterial pathogens. Mol. Plant Microbe Int. 22: 1227-1238.

Wei CM, Wang L, Yang YX, Chen ZX, et al. (2010). Identification of an $S_{5}{ }^{n}$ allele in Oryza rufipogon Griff. and its effect on embryo sac fertility. Chin. Sci. Bull. 55: 1255-1262.

Wing RA, Ammiraju JS, Luo M, Kim HR, et al. (2005). The Oryza map alignment project: the golden path to unlocking the genetic potential of wild rice species. Plant Mol. Biol. 59: 53-62. 
Wu X, Shiroto Y, Kishitani S, Ito $\mathrm{Y}$, et al. (2009). Enhanced heat and drought tolerance in transgenic rice seedlings overexpressing OsWRKY11 under the control of HSP101 promoter. Plant Cell Rep. 28: 21-30

Yamaguchi-Shinozaki K, Koizumi M, Urao S and Shinozaki K (1992). Molecular cloning and characterization of $9 \mathrm{cDNAs}$ for genes that are responsive to desiccation in Arabidopsis thaliana: sequence analysis of one cDNA clone that encodes a putative transmembrane channel protein. Plant Cell Physiol. 33: 217-224.

Yan H, Hu H, Fu Q, Yu H, et al. (1996). Morphological and cytogenetical studies of the hybrids between Oryza sativa and Oryza officinalis. Chin. J. Rice Sci. 10: 138-142.

Yang YX, Li YH, Tong JF, Shahid MQ et al. (2012). Wide-compatibility gene $S_{5}{ }^{n}$ exploited by functional molecular markers and its effect on fertility of intersubspecific rice hybrids. Crop Sci. 52: 669-675.

Zhong DB, Luo LJ and Ying CS (1995). Utilization of wild rice in cultivar rice breeding. Seed 14: 25-29. 